\title{
Profissionais de Saúde Mental: Estresse, Enfrentamento e Qualidade de Vida ${ }^{1}$
}

\author{
Ana Flávia de Oliveira Santos \\ Carmen Lúcia Cardoso ${ }^{2}$ \\ Universidade de São Paulo (Ribeirão Preto)
}

\begin{abstract}
RESUMO - Objetivou-se avaliar estresse, enfrentamento e qualidade de vida de profissionais de serviços substitutivos de saúde mental. Participaram 25 profissionais. Aplicaram-se os seguintes instrumentos: Inventário de Sintomas de Stress para Adultos, Inventário de Estratégias de Enfrentamento e Escala de Qualidade de Vida. Os resultados apontaram que 36\% dos profissionais apresentavam estresse, maior uso da estratégia de suporte social e maior satisfação com o domínio social da qualidade de vida. Profissionais com presença de estresse utilizaram mais as estratégias de afastamento e fuga-esquiva. Conclui-se que os profissionais de saúde mental, sob estresse, empregam mais estratégias de enfrentamento centradas na emoção. É necessária a elaboração e o desenvolvimento de intervenções que auxiliem esses profissionais no manejo de situações estressantes.
\end{abstract}

Palavras-chave: estresse; qualidade de vida; saúde mental.

\section{Mental Health Professionals: Stress, Coping and Quality of Life}

\begin{abstract}
The purpose of the study was to assess stress, coping, and quality of life of professionals in substitutive mental health services. Twenty-five professionals participated in this study. The following instruments were employed: Stress Symptoms Inventory for Adults, Coping Strategies Inventory and the Quality of Life Scale. The results showed that $36 \%$ of the professionals presented stress, greater use of the social support strategy, and greater satisfaction with the social domain regarding quality of life. Professionals with stress indicators used more withdrawal and escape-avoidance strategies. In conclusion, mental health professionals under stress make more use of coping strategies focused on emotions. The elaboration and development of interventions to assist these professionals in handling stressful situations are needed.
\end{abstract}

Keywords: stress; quality of life; mental health.

O estresse foi definido por Selye (1956) como "Síndrome Geral de Adaptação", referindo-se à quebra da homeostase interna frente a um evento estressor, o que exige do indivíduo esforço em termos de adaptação. Seu processo de desencadeamento se baseou em um modelo trifásico, a saber: fase de alerta, fase de resistência e fase de exaustão. Uma vez que a adaptabilidade do organismo é finita, se o estressor não for suprimido ou se o indivíduo não souber administrá-lo, poderá chegar à exaustão física e psicológica, momento em que as doenças se manifestam, podendo ser fatais (Selye, 1983). Assim, o estresse não causa doenças, apenas atua como um facilitador para o seu surgimento (Lipp \& Malagris, 1998).

No contexto do trabalho, destaca-se a síndrome de burnout, uma síndrome psicológica em resposta aos estressores interpessoais crônicos no trabalho, decorrente de uma má adaptação a um trabalho estressante, prolongado e com elevada carga tensional. O indivíduo que experiencia o burnout pode apresentar sentimentos de frustração quanto a si e a seu trabalho, o que envolve esgotamento emocional, desperso-

1 Pesquisa financiada pela Fundação de Amparo à Pesquisa do Estado de São Paulo - FAPESP (Bolsa de Iniciação Científica) - Número do processo: 2005/04662-1.

2 Endereço para correspondência: Departamento de Psicologia e Educação, Faculdade de Filosofia, Ciências e Letras de Ribeirão Preto, Universidade de São Paulo. Av. Bandeirantes, 3900. Ribeirão Preto, SP. CEP 14040-901. E-mail: carmen@ffclrp.usp.br. nalização e baixa realização pessoal no trabalho (Maslach, Schaufeli \& Leiter, 2001).

Conforme apontam Lazarus e Folkman (1984), a percepção e a interpretação do indivíduo ante uma dada situação são de demasiada importância para o desencadeamento da reação de estresse, ganhando destaque a questão do coping (enfrentamento), definido como esforços cognitivos e comportamentais que visam reduzir a situação avaliada como excessiva ou estressora ao indivíduo.

Segundo Selye (1983), pode-se lidar com situações estressantes de diferentes formas, a saber: a partir da remoção dos estressores desnecessários à vida; evitando-se que eventos neutros se tornem estressores; desenvolvendo habilidades de enfrentamento a condições adversas; por meio de relaxamento e distração.

Os profissionais de saúde mental, por realizarem o seu trabalho em um ambiente com elevada demanda emocional, encontram-se vulneráveis à tensão psicológica e à exaustão emocional, estando susceptíveis ao desenvolvimento do estresse e do burnout (Moore \& Cooper, 1996). A capacidade de enfrentamento dos profissionais face a situações estressoras apresenta impacto direto sobre sua qualidade de vida. Burrows e McGrath (2000) afirmam que, para se lidar com o estresse, estratégias efetivas de coping devem ser desenvolvidas e incorporadas em programas de treinamento de profissionais de saúde mental. 
Considerando que o estresse se situa como um importante agente facilitador no estabelecimento de doenças, faz-se necessário o desenvolvimento de estratégias de enfrentamento para evitar consequências maléficas para a qualidade de vida dos indivíduos (Lipp, 1996). Conforme indicam Lipp e Malagris (1998), o nível de qualidade de vida, em quaisquer de suas áreas, estados de bem-estar mental, físico e social, encontra-se profundamente afetado pelo grau de estresse que a pessoa apresenta.

Segundo a Organização Mundial da Saúde (OMS), a qualidade de vida é definida como "a percepção do indivíduo de sua posição na vida no contexto da cultura e sistema de valores nos quais ele vive e em relação aos seus objetivos, expectativas, padrões e preocupações" (WHOQOL Group, conforme citado por Fleck \& cols., 2000, p. 179). Assim, mostra-se de fundamental importância considerar a percepção subjetiva quanto à satisfação do indivíduo em relação a diferentes aspectos da qualidade de vida, como seus componentes físico, psicológico, social, cultural e espiritual.

O presente trabalho tem por objetivo avaliar a manifestação de estresse e a qualidade de vida de profissionais de saúde mental de serviços substitutivos caracterizados pelo modo psicossocial de atenção, bem como as estratégias de enfrentamento utilizadas ante a situação cotidiana de contato com usuário em sofrimento mental.

\section{Método}

\section{Participantes}

O estudo foi realizado com 25 trabalhadores de saúde mental contratados por serviços substitutivos ambulatoriais e de internação parcial de saúde mental de uma cidade do interior de São Paulo. O presente trabalho foi aprovado pelo Comitê de Ética em Pesquisa, protocolo nº167/CEP/CSE-FMRP-USP.

O perfil sócio-demográfico dos participantes se encontra na Tabela 1.

\section{Instrumentos}

Para a coleta de dados, foram utilizados os seguintes instrumentos:

(a) Inventário de Stress para Adultos de Lipp - ISSL: validado por Lipp (2000), permite identificar a presença de sintomas de estresse, os tipos de sintomatologia - somática ou psicológica - e a fase de estresse em que o indivíduo se encontra;

(b) Inventário de Estratégias de Coping de Folkman e Lazarus: validado para o Brasil por Savoia (1999), objetiva avaliar as estratégias de enfrentamento utilizadas para lidar com situações estressoras específicas. Aborda oito fatores como estratégias de enfrentamento, a saber: confronto, afastamento, autocontrole, suporte social, aceitação de responsabilidade, fuga e esquiva, resolução de problemas e reavaliação positiva;
Tabela 1. Caracterização em frequência simples (f) e porcentagem (\%) dos participantes $(\mathrm{n}=25)$ em função das variáveis sociodemográficas.

\begin{tabular}{|c|c|c|c|}
\hline Variável & Especificação & $F$ & $\%$ \\
\hline \multirow[t]{2}{*}{ Sexo } & Feminino & 19 & $76,00 \%$ \\
\hline & Masculino & 6 & $24,00 \%$ \\
\hline \multirow[t]{5}{*}{$\begin{array}{l}\text { Faixa etária } \\
\text { (anos) }\end{array}$} & $20-30$ anos & 4 & $16,00 \%$ \\
\hline & $31-40$ anos & 5 & $20,00 \%$ \\
\hline & $41-50$ anos & 9 & $36,00 \%$ \\
\hline & $51-60$ anos & 6 & $24,00 \%$ \\
\hline & $61-70$ anos & 1 & $4,00 \%$ \\
\hline Nível & II grau completo & 4 & $16,00 \%$ \\
\hline \multirow[t]{4}{*}{ educacional } & III grau incompleto & 3 & $12,00 \%$ \\
\hline & III grau completo & 9 & $36,00 \%$ \\
\hline & Pós-graduação incompleta & 3 & $12,00 \%$ \\
\hline & Pós-graduação completa & 6 & $24,00 \%$ \\
\hline \multirow[t]{3}{*}{ Estado civil } & Solteiro & 7 & $28,00 \%$ \\
\hline & Casado & 13 & $52,00 \%$ \\
\hline & Separado/divorciado & 5 & $20,00 \%$ \\
\hline \multirow[t]{4}{*}{$\begin{array}{l}\text { Número de } \\
\text { filhos }\end{array}$} & 0 & 9 & $36,00 \%$ \\
\hline & 1 & 6 & $24,00 \%$ \\
\hline & 2 & 8 & $32,00 \%$ \\
\hline & $>4$ & 1 & $4,00 \%$ \\
\hline \multirow[t]{5}{*}{ Religião } & Católica & 7 & $28,00 \%$ \\
\hline & Evangélica & 6 & $24,00 \%$ \\
\hline & Espírita & 6 & $24,00 \%$ \\
\hline & Outra & 2 & $8,00 \%$ \\
\hline & Não tem & 4 & $16,00 \%$ \\
\hline \multirow[t]{2}{*}{ Praticante } & Sim & 14 & $66,67 \%$ \\
\hline & Não & 7 & $33,33 \%$ \\
\hline \multirow{6}{*}{$\begin{array}{l}\text { Renda pessoal } \\
\text { (reais) }\end{array}$} & $100-1000$ & 5 & $20,00 \%$ \\
\hline & $1100-2000$ & 10 & $40,00 \%$ \\
\hline & $2100-3000$ & 4 & $16,00 \%$ \\
\hline & $3100-4000$ & 2 & $8,00 \%$ \\
\hline & $4100-5000$ & 3 & $12,00 \%$ \\
\hline & $5100-6000$ & 1 & $4,00 \%$ \\
\hline
\end{tabular}

(c) Escala de Qualidade de Vida da OMS (WHOQOL-Bref): traduzida e validada para o português por Fleck e cols. (2000), objetiva avaliar a qualidade de vida do indivíduo em quatro domínios: físico, psicológico, relações sociais e meio ambiente;

(d) Roteiro Complementar: elaborado pela pesquisadora, possibilita identificar os aspectos sciodemográficos dos trabalhadores (15 itens).

\section{Procedimento}

Primeiramente, foi ressaltado o caráter voluntário da colaboração, esclarecendo-se as condições de participação por meio do Termo de Consentimento Livre e Esclarecido, o qual foi lido e assinado pelos participantes. Os participantes responderam aos instrumentos na presença da pesquisadora, 
que esteve disponível para eventuais esclarecimentos. Os questionários foram aplicados e cotados segundo suas proposições técnicas. Procedeu-se a tratamento estatístico não-paramétrico dos dados, a partir do Teste de Mann-Whitney e do Coeficiente de Correlação de Postos de Spearman, adotando-se valores de $\mathrm{p} \leq 0,05$.

\section{Resultados}

Verificou-se que, a partir dos resultados obtidos pelo ISSL, 36,0\% dos participantes apresentaram indicadores de presença de manifestação de estresse. Desses, 88,9\% encontravam-se na fase de resistência e $11,1 \%$, na de exaustão. Quanto à sintomatologia apresentada, 66,7\% referiram predominantemente sintomas físicos.

Em relação às estratégias empregadas frente à situação de contato cotidiano com usuário em sofrimento mental, obtiveram-se os resultados apresentados na Tabela 2. Conforme se verifica pela distribuição das médias de respostas para as estratégias de enfrentamento utilizadas pelos profissionais de saúde mental, ocorreu maior uso das estratégias de suporte social $(M=1,75)$, resolução de problemas $(M=1,64)$ e autocontrole $(\mathrm{M}=1,54)$. As estratégias de afastamento $(\mathrm{M}=0,95)$ e de confronto $(\mathrm{M}=0,79)$ foram as menos utilizadas.

Os escores obtidos para cada domínio da qualidade de vida, de acordo com o WHOQOL-Bref, apresentam-se na Tabela 3. Verificou-se maior satisfação com o domínio social da qualidade de vida (Med=75,0).

Quanto à qualidade de vida geral, tem-se que $72 \%$ dos participantes avaliam sua qualidade de vida como sendo boa ou muito boa; quanto à própria saúde, $44 \%$ dos participantes estão nem satisfeitos nem insatisfeitos e $40 \%$, satisfeitos ou muito satisfeitos.

Em relação à estatística inferencial, observou-se média superior de uso da estratégia de suporte social para a variável sexo feminino, quando comparado ao sexo masculino ( $p=0,05$; Teste de Mann-Whitney).

No que diz respeito à escolaridade, foi encontrada média significativamente superior de uso da estratégia de resolução de problemas entre participantes com ensino superior ou pós-graduação, completa ou incompleta, quando comparado com participantes com ensino médio completo ou ensino superior incompleto ( $\mathrm{p}=0,03$; Teste de Mann-Whitney).
Obteve-se correlação negativa, de acordo com o Coeficiente de Correlação de Postos de Spearman, entre tempo de serviço em saúde mental e uso da estratégia de confronto $(r=-0,42 ; p=0,03)$, uso da estratégia de afastamento $(r=-0,52$; $\mathrm{p}=0,007)$, uso da estratégia de aceitação da responsabilidade $(\mathrm{r}=-0,46 ; \mathrm{p}=0,02)$, uso da estratégia de fuga-esquiva $(\mathrm{r}=-0,40$; $\mathrm{p}=0,04)$, uso de resolução de problemas $(\mathrm{r}=-0,44 ; \mathrm{p}=0,02)$ e uso da estratégia de reavaliação positiva $(\mathrm{r}=-0,42 ; \mathrm{p}=0,03)$. Esses dados indicam que, conforme aumenta o tempo de serviço em saúde mental, menor é o uso das respectivas estratégias. Não foram encontradas correlações entre os fatores de enfrentamento autocontrole e suporte social e a variável tempo de serviço.

Verificou-se correlação negativa entre tempo de serviço no local pesquisado e uso da estratégia de reavaliação positiva $(\mathrm{r}=-0,38 ; \mathrm{p}=0,05$; Coeficiente de Correlação de Postos de Spearman), ou seja, com o aumento do tempo de serviço no local, menos se faz o uso da estratégia de reavaliação positiva.

A análise dos fatores de enfrentamento obtidos pelo Inventário de Estratégias de Coping em função da presença e ausência de indicadores de estresse obtidos pelo ISSL indicou médias superiores dos fatores afastamento $(p=0,03$; Teste de Mann-Whitney) e fuga-esquiva ( $p=0,03$; Teste de Mann-Whitney) para os profissionais com presença de indicadores de estresse, quando comparados com aqueles com ausência de estresse. Isso sugere que, em relação à sobrecarga frente ao contato cotidiano com usuários em sofrimento mental, há maior uso das estratégias de afastamento e fuga-esquiva entre os participantes que apresentam indicadores de presença de estresse.

Observou-se que os domínios psicológico e social da qualidade de vida apresentaram médias significativamente superiores associadas à ausência de indicadores de estresse, quando comparados com a presença de estresse $(\mathrm{p}=0,05$ e $\mathrm{p}=0,01$, respectivamente; Teste de Mann Whitney). Não foram encontradas diferenças estatisticamente significativas nas análises dos domínios físico e meio ambiente com indicadores de estresse.

A média de satisfação com o domínio meio ambiente da escala de qualidade de vida do WHOQOL-Bref foi significativamente superior para participantes com ensino superior ou com pós-graduação, completa ou incompleta, quando comparado ao ensino médio completo ou ensino superior incompleto ( $\mathrm{p}=0,04$; Teste de Mann-Whitney).

Em relação aos domínios físico e psicológico (WHOQOL-Bref), foram encontradas médias significati-

Tabela 2. Mediana, média, desvio padrão e intervalo mínimo-máximo obtidos para cada fator do Inventário de Estratégias de Enfrentamento (Coping) entre os profissionais de saúde mental dos três serviços pesquisados, de acordo com as proposições de Guido (2003).

\begin{tabular}{lcccc}
\hline Fatores do Inventário & Mediana & Média & Desvio Padrão & $\begin{array}{c}\text { Intervalo mínimo- } \\
\text {-máximo }\end{array}$ \\
\hline Confronto & 0,67 & $\mathbf{0 , 7 9}$ & 0,37 & $0,16-1,83$ \\
Afastamento & 0,86 & 0,95 & 0,50 & $0,29-2,29$ \\
Autocontrole & 1,6 & 1,54 & 0,52 & $0,80-2,60$ \\
Suporte social & 1,83 & $\mathbf{1 , 7 5}$ & 0,69 & $0,83-2,67$ \\
Aceitação de responsabilidade & 1,71 & 1,51 & 0,58 & $0,29-2,29$ \\
Fuga e esquiva & 1,0 & 1,18 & 0,80 & $0,00-2,50$ \\
Resolução de problemas & 1,75 & 1,64 & 0,70 & $0,25-2,75$ \\
Reavaliação positiva & 1,56 & 1,48 & 0,53 & $0,44-2,56$ \\
\hline
\end{tabular}


Tabela 3. Mediana, média, desvio padrão e intervalo mínimo-máximo obtidos para os quatro domínios do instrumento WHOQOL-Bref.

\begin{tabular}{|c|c|c|c|c|}
\hline Domínio & $\begin{array}{c}\text { Intervalo mínimo- } \\
\text {-máximo }\end{array}$ & Mediana & Média & Desvio Padrão \\
\hline Físico & $35,71-89,29$ & 71,43 & 69,14 & 12,62 \\
\hline Psicológico & $37,50-83,33$ & 58,33 & 63,17 & 12,99 \\
\hline Relações sociais & $25,00-100,00$ & 75,0 & 65,33 & 20,51 \\
\hline Meio ambiente & $28,13-84,38$ & 56,25 & 55,75 & 12,29 \\
\hline
\end{tabular}

vamente superiores de satisfação para os profissionais que têm alguma religião, quando comparados com os que não possuem religião ( $\mathrm{p}=0,03$ e $\mathrm{p}=0,04$; Teste de Mann-Whitney).

Encontraram-se médias significativamente superiores de satisfação nos domínios social e meio ambiente para aqueles profissionais que possuem até seis horas de contato com os usuários, quando comparado a mais de seis horas de contato ( $p=0,05$; Teste de Mann-Whitney).

Correlações positivas entre o domínio social e a variável renda $(r=0,39 ; p=0,05$; Coeficiente de Correlação de Postos de Spearman), bem como entre o domínio meio ambiente e a variável renda $(\mathrm{r}=0,46 ; \mathrm{p}=0,01$; Coeficiente de Correlação de Postos de Spearman) foram observadas, indicando que, conforme aumenta a satisfação nesses domínios, maior é a renda.

\section{Discussão}

Uma parcela considerável dos profissionais de saúde mental estudados apresenta manifestações de estresse, estando quase sua totalidade na fase de resistência. Nesse momento, o estresse já se encontra instalado, exigindo esforços na tentativa de restabelecer o equilíbrio interno. A energia para adaptação é finita (Selye, 1983), havendo a necessidade de que esses profissionais desenvolvam estratégias para melhor manejar os eventos estressores, evitando um maior enfraquecimento físico e o posterior desenvolvimento de doenças. Os indicadores de estresse apresentados são predominantemente de natureza física, como tensão muscular, taquicardia, dores de cabeça, desgaste físico constante, entre outros. Tais sintomas podem interferir negativamente na disposição do indivíduo, inclusive em suas atividades laborais voltadas ao cuidado do usuário, demandando o desenvolvimento de estratégias mais adaptativas por esses profissionais.

A respeito do enfrentamento dos profissionais em relação à sobrecarga decorrente do contato cotidiano com usuários em sofrimento mental, dado obtido pelo Inventário de Estratégias de Coping, houve uma maior utilização de estratégias de suporte social, seguido por resolução de problemas e autocontrole, sendo menos utilizadas as estratégias de afastamento e de confronto. De modo geral, esses resultados apontam para o maior uso de estratégias ligadas à disposição para a busca de apoio e de ajuda no relacionamento com o outro (suporte social), indicando ser esta a forma mais utilizada para enfrentar tal situação. Esforços para planejar ações e caminhar em direção a resolver problemas relacionados ao contato com os usuários (resolução de problemas), bem como para a regulação de sentimentos e ações frente a essas situações de modo a não agir precipitadamente ou por impulso (autocontrole) se encontram entre os mecanismos mais utilizados.

Segundo Acker (1999), o suporte social atua como um importante fator para que os assistentes sociais possam enfrentar as situações estressantes no trabalho e constitui um fator de satisfação entre os trabalhadores. Maslach e cols. (2001) afirmam que a falta de suporte social constitui um preditor para o burnout. O suporte social, dessa forma, serviria como um mediador na relação entre vivência do trabalhador em relação aos estressores do trabalho e burnout.

A associação encontrada entre uso da estratégia de suporte social e a variável sexo feminino sugere uma influência da cultura que favorece a mulher a ter maior facilidade para lidar com o sofrimento e falar sobre sentimentos, apresentando uma disponibilidade e abertura maiores para o compartilhamento de experiência voltada à obtenção de suporte social frente às situações adversas (Bandeira \& Oliveira, 1998).

As estratégias menos utilizadas no contato com os usuários se relacionam à procura pelo distanciamento da situação ou sua não-resolução (afastamento) ou o manejo por meio de esforços agressivos contrários ao contato com os usuários (confronto), indicando serem essas estratégias menos adaptativas à situação que vivenciam. A menor utilização das estratégias de afastamento e confronto em relação à sobrecarga gerada pelo contato com os usuários com dificuldades emocionais pode apontar para uma atitude favorável ao contato com esses usuários.

Em relação às estratégias de enfrentamento, destaca-se que foi encontrada associação estatisticamente significativa entre os fatores de afastamento e de fuga-esquiva e a presença de indicadores de estresse avaliados por meio do ISSL. Maior uso das estratégias de afastamento e fuga-esquiva entre os participantes que apresentam indicadores de presença de estresse indica possivelmente uma forma de assegurar a preservação da própria saúde mental. Essa forma se constitui na utilização de uma estratégia de defesa pessoal que afeta diretamente a qualidade do trabalho, uma vez que a atuação nessa área pressupõe cuidado direto ao usuário, demandando uma aproximação técnica e afetiva por parte desses profissionais. Além disto, possibilita refletir que, apesar do caráter defensivo dessas estratégias em relação ao trabalho, as mesmas não são suficientes para lidar com o estresse e podem inclusive colaborar para a sua manutenção. Vale ressaltar que o uso de tais estratégias por esses profissionais pode dificultar o estabelecimento de vínculo com o usuário e o cuidado integral do mesmo, disponibilidades internas tão requeridas pelo modelo psicossocial em prática na saúde mental.

Quanto ao uso das estratégias de confronto, afastamento e fuga-esquiva em relação ao tempo de serviço em saúde 
mental, foi encontrada correlação negativa, indicando que, conforme aumenta o tempo de serviço em saúde mental, diminui-se o uso dessas estratégias. Esse resultado aponta para a importância da experiência para a aprendizagem e o emprego de estratégias mais adaptativas no contexto de trabalho, reforçando a necessidade de acompanhamento do profissional, em particular o iniciante, para o treinamento de técnicas apropriadas e o desenvolvimento de habilidades para o manejo de situações estressantes.

Na mesma direção, foi encontrada correlação negativa entre a variável tempo de serviço no local pesquisado e uso da estratégia de reavaliação positiva, sinalizando para a influência do trabalho, especificamente no que se refere ao ambiente e seus correlatos, como fator que tende a levar os profissionais a empreenderem menos esforços de ressignificação da situação e de busca por sua mudança. Guido (2003), verificando as estratégias de coping entre enfermeiros de centro cirúrgico e recuperação anestésica, constatou correlação semelhante, indicando uma relação inversa entre menor uso da estratégia de reavaliação positiva e maior tempo de serviço no hospital.

Nesse sentido, cabe considerar o papel da supervisão clínica e de estratégias de ajuda adotados pelas instituições como forma de apoio organizacional ao trabalhador. Reid e cols. (1999) apontam que a supervisão consiste principalmente em prover suporte geral em relação ao manejo clínico, com espaço restrito à discussão de casos clínicos, não oferecendo ambiente acolhedor para discussão a respeito da sobrecarga emocional referente ao cuidado dos usuários em sofrimento mental, estratégias de redução de estresse ou mesmo discussões sobre carreira e desenvolvimento profissional. Os referidos autores indicam a necessidade de se maximizar a contribuição advinda da colaboração dos colegas de trabalho como potenciais recursos informais de suporte, propiciando eventos sociais e atividades estruturadas, tais como realização de grupos estruturados para a equipe toda, contando com uma postura ativa do facilitador; promoção de eventos em que os membros da equipe possam se encontrar, fora do trabalho, para realizar atividades diversas, ou simplesmente encontros para almoços. Fothergill, Edwards e Burnard (2004) apontam que estratégias de enfrentamento mais efetivas devem ser implementadas a nível organizacional e individual.

As correlações negativas entre o uso das estratégias de se sentir culpado pela situação (aceitação da responsabilidade), resolução de problemas e de reflexão, flexibilização e ressignificação da experiência (reavaliação positiva) e a variável tempo de serviço em saúde mental indicaram que, com o aumento da experiência nesse campo profissional, esses profissionais utilizam menos essas estratégias de enfrentamento. Assim, frente à sobrecarga do contato com os usuários, são menos utilizadas as estratégias de culpabilização e crítica de si. Observa-se também menor busca de planejar e executar um plano voltado à resolução do problema e uma menor capacidade de flexibilização e ressignificação da situação, com menor uso da criatividade para se lidar com esta, minimizando a possibilidade de se sair bem da experiência e crescendo a partir dela. Pode-se supor, assim, certa acomodação em relação à situação e passividade no enfrentamento de situações avaliadas como estressoras.
A associação significativa encontrada entre uso da estratégia de resolução de problemas e participantes com ensino superior ou pós-graduação sinaliza para a relevância do papel da aprendizagem continuada e da experiência profissional para o desenvolvimento de estratégias mais adaptativas no ambiente de trabalho. Profissionais que procuram o aprimoramento de suas habilidades e conhecimentos e que persistem na busca pela instrumentalização necessária ao seu trabalho podem agregar ao cuidado oferecido o caráter de apoio e atenção ao usuário, estando, pois, consoantes com o modelo psicossocial pretendido.

Quanto aos índices de satisfação com a qualidade de vida, os escores obtidos pelos participantes da pesquisa foram acima da média para todos os domínios avaliados pelo WHOQOL-Bref, a saber: meio ambiente, físico, psicológico e social. Baseando-se nos resultados obtidos, o grupo apresentou maior satisfação em relação ao domínio social, seguido pela satisfação no domínio físico, psicológico e meio ambiente, respectivamente. O domínio social engloba os seguintes aspectos: relações pessoais, suporte, apoio social e atividade sexual. Esse resultado indica que esses profissionais vivenciam satisfação mais acentuada no campo das relações sociais, apontando para a significativa relevância da rede social de apoio, bem como da consideração do contexto social do trabalho.

Nesse sentido, pode-se sinalizar a congruência entre maior satisfação relativa às relações sociais e uso da estratégia de suporte social, o que indica a busca, nas relações, de apoio às dificuldades vivenciadas. $\mathrm{O}$ apoio social pode tanto ser advindo de familiares, amigos ou mesmo dos próprios colegas de trabalho. Conforme apontado anteriormente, tomar medidas de criação de espaços de suporte ao profissional pode ser, então, efetivo tanto para a organização quanto para a sociedade, uma vez que auxilia o trabalhador no enfrentamento de suas dificuldades.

A correlação estatisticamente significativa entre os domínios psicológico e social da qualidade de vida e a ausência de indicadores de estresse sugere que as pessoas mais satisfeitas nos campos da aceitação, satisfação e crenças pessoais e do suporte social não apresentaram indicadores de estresse, reafirmando a importância do apoio para a satisfação e preservação de sua saúde.

Os profissionais que possuem até seis horas de contato diário com os usuários apresentaram significativamente maior satisfação no domínio social e no domínio ambiente. Uma vez que horas de contato com usuários muitas vezes é significada como carga horária de trabalho, esses resultados podem sugerir que profissionais com carga reduzida de serviço em saúde mental possuem maior disponibilidade em ter acesso a oportunidades de recreação e possibilidades de estabelecimento de relacionamentos interpessoais extratrabalho, o que poderia acarretar satisfação nos domínios social e meio ambiente mais acentuada.

Os participantes com escolaridade equivalente ao ensino superior ou pós-graduação apresentaram significativamente maior satisfação com o domínio meio ambiente. Esse resultado sugere que, para os profissionais com uma escolaridade mais elevada, o trabalho pode oferecer acesso a diferentes recursos em favor da vida (melhores condições de moradia, acesso a lazer, entre outras) e também favorece a assunção de atividades de maior retorno financeiro. Nessa mesma direção, 
foi encontrada correlação positiva em relação aos domínios social e meio ambiente e a variável renda, demonstrando que participantes com maior satisfação nesses domínios possuem maior renda. Pode-se inferir que uma faixa de renda superior também favoreça o acesso a lazer, o que pode facilitar o estabelecimento de relações interpessoais para além do trabalho. Além disso, maiores recursos financeiros propiciam acesso a serviços de saúde e oportunidade de moradia mais consoante às aspirações dos participantes.

Como aponta Kanters (conforme citado por Kluthcovsky, 2005), o lazer pode fornecer oportunidades de interação social e auxiliar no sentimento de bem-estar e no desenvolvimento do potencial individual, trazendo benefícios para a saúde e para o controle do estresse.

Em relação aos domínios físico e psicológico da qualidade de vida, foram encontradas relações significativas de satisfação para os profissionais que têm religião. Na percepção desses profissionais, a religião pode se configurar como suporte e apoio sociais e, assim, contribuir para uma maior qualidade de vida. O domínio psicológico apresenta a faceta espiritualidade, religião e crenças pessoais, buscando avaliar o sentido que o indivíduo encontra na vida.

A partir dos resultados obtidos, apreende-se a importância do investimento no cuidado ao cuidador, propiciando as condições necessárias para o desempenho do trabalho em saúde mental.

Aponta-se o número de participantes que compõem a amostra $(n=25)$ como uma limitação do presente estudo. Contudo, uma vez que foi desenvolvido no contexto de três serviços substitutivos, a colaboração de $73,5 \%$ do total de profissionais indica uma amostra representativa das instituições existentes

Os resultados sinalizam a necessidade de um acompanhamento efetivo junto aos trabalhadores, fornecendo-lhes cuidado por meio da criação e manutenção de espaços para supervisão, tutoria e grupos de reflexão. Ainda, investimentos na forma de programas de formação continuada devem ser incentivados, ajudando os trabalhadores em sua qualificação profissional, resgatando-se a necessidade do caráter permanente da aprendizagem. Ressalta-se ainda a importante contribuição do fator de apoio advindo dos relacionamentos intra e/ou extratrabalho para que os profissionais possam enfrentar as situações estressantes do contexto ocupacional.

A avaliação de aspectos psicossociais do trabalho junto ao jovem profissional, bem como o acompanhamento em suas atividades diárias, buscando identificar as dificuldades vivenciadas e os seus recursos potenciais poderiam, ainda, oferecer subsídios para a estruturação de programas de formação continuada que auxiliem na adaptação do profissional, refletindo, por conseguinte, numa boa qualidade da assistência.

\section{Referências}

Acker, G. M. (1999). The impact of client's mental illness on social workers' job satisfaction and burnout. Health Social Work, 24, 112-9.
Bandeira, L., \& Oliveira, E. M. (1998). Representações de gênero e moralidade na prática profissional da enfermagem. Revista Brasileira de Enfermagem, 51, 677-696.

Burrows, D., \& McGrath, C. (2000). Stress and mental health professionals. Stress Medicine, 16, 269-270.

Fleck, M. P. A., Louzada, S., Xavier, M., Chachamovich, E., Vieira, G., Santos, L., \& Pinzon, V. (2000). Aplicação da versão em português do instrumento WHOQOL-Bref. Revista Saúde Pública, 34, 178-83.

Fothergill, A., Edwards, D., \& Burnard, P. (2004). Stress, burnout, coping and stress management in psychiatrists: Findings from a systematic review. International Journal of Social Psychiatry, 50, 54-65.

Guido, L. A. (2003). Stress e coping entre enfermeiros de centro cirúrgico e recuperação anestésica. Tese de Doutorado, Universidade de São Paulo, Ribeirão Preto.

Kluthcovsky, A. C. G.C. (2005). Qualidade de vida dos agentes comunitários de saúde de um município do interior do Paraná. Dissertação de Mestrado, Universidade de São Paulo, Ribeirão Preto.

Lazarus, R. S., \& Folkman, S. (1984). Stress, appraisal and coping. New York: Sringer.

Lipp, M. E. N. (1996). Stress: conceitos básicos. Em M. E. N. Lipp (Ed.), Pesquisas sobre stress no Brasil-saúde, ocupações e grupos de risco (pp. 17-31). São Paulo: Papirus.

Lipp, M. E. N. (2000). Inventário de Sintomas de Stress para Adultos de Lipp (ISSL) - manual. São Paulo: Casa do Psicólogo.

Lipp, M. E. N., \& Malagris, L. N. (1998). Manejo de estresse. Em B. Rangé (Ed.), Psicoterapia comportamental e cognitiva (pp. 279-292). São Paulo: Editorial Psy.

Maslach, C., Schaufeli, W. B., \& Leiter, M. P. (2001). Job burnout. Annual Review Psychology, 52, 397-422.

Moore, K. A., \& Cooper, C. L. (1996). Stress in mental health professionals: A theoretical overview. International Journal of Social Psychiatry, 42, 82-89.

Reid, Y, Johnson, S., Morant, N., Kuipers, E., Szmukle, G., Bebbington, P., Thornicroft, G., \& Prosser, D. (1999). Improving support for mental health staff: A qualitative study. Social Psychiatry and Psychiatric Epidemiology, 34, 309-315.

Savoia, M. G. (1999). Escalas de eventos vitais e de estratégias de enfrentamento (Coping). Revista de Psiquiatria Clínica, 26, 57-67.

Selye, H. (1956). The stress of life. New York: Longmans.

Selye, H. (1983). The stress concept: Past, present and future. Em C. L. Cooper (Ed.), Stress research: Issues for the eighties (pp. 1-20). USA: John Wiley \& Sons. 